\title{
AN IRON K COMPONENT TO THE ULTRAFAST OUTFLOW IN NGC 1313 X-1
}

\author{
D. J. Walton ${ }^{1,2}$, M. J. Middleton ${ }^{3}$, C. Pinto $^{3}$, A. C. Fabian ${ }^{3}$, M. Bachetti ${ }^{4}$, D. Barret ${ }^{5,6}$, \\ M. Brightman ${ }^{2}$, F. Fuerst ${ }^{2}$, F. A. Harrison $^{2}$, J. M. Miller ${ }^{7}$, and D. Stern ${ }^{1}$ \\ ${ }^{1}$ Jet Propulsion Laboratory, California Institute of Technology, Pasadena, CA 91109, USA \\ ${ }^{2}$ Space Radiation Laboratory, California Institute of Technology, Pasadena, CA 91125, USA \\ ${ }^{3}$ Institute of Astronomy, University of Cambridge, Madingley Road, Cambridge CB3 OHA, UK \\ ${ }^{4}$ INAF/Osservatorio Astronomico di Cagliari, via della Scienza 5, I-09047 Selargius (CA), Italy \\ ${ }^{5}$ Universite de Toulouse, UPS-OMP, IRAP, Toulouse, France \\ ${ }^{6}$ CNRS; IRAP; 9 Av. colonel Roche, BP 44346, F-31028 Toulouse cedex 4, France \\ ${ }^{7}$ Department of Astronomy, University of Michigan, 1085 S. University Avenue, Ann Arbor, MI 49109-1107, USA \\ Received 2016 June 30; revised 2016 July 6; accepted 2016 July 6; published 2016 July 29
}

\begin{abstract}
We present the detection of an absorption feature at $E=8.77_{-0.06}^{+0.05} \mathrm{keV}$ in the combined X-ray spectrum of the ultraluminous X-ray source NGC 1313 X-1 observed with XMM-Newton and NuSTAR, significant at the $3 \sigma$ level. If associated with blueshifted ionized iron, the implied outflow velocity is $\sim 0.2 c$ for Fe XXVI, or $\sim 0.25 c$ for Fe XXV. These velocities are similar to the ultrafast outflow seen in absorption recently discovered in this source at lower energies by XMM-Newton, and we therefore conclude that this is an iron component to the same outflow. Photoionization modeling marginally prefers the Fe XXV solution, but in either case the outflow properties appear to be extreme, potentially supporting a super-Eddington hypothesis for NGC $1313 \mathrm{X}-1$.
\end{abstract}

Key words: black hole physics - X-rays: binaries - X-rays: individual (NGC 1313 X-1)

\section{INTRODUCTION}

Ultraluminous X-ray sources (ULXs) are variable, offnuclear point sources in nearby galaxies with $\mathrm{X}$-ray luminosities $L_{\mathrm{X}} \geqslant 10^{39} \mathrm{erg} \mathrm{s}^{-1}$ (Swartz et al. 2004; Walton et al. 2011). The brighter members of this population have luminosities that significantly (factors of 10 or more) exceed the Eddington limit for the $\sim 10 M_{\odot}$ stellar-remnant black holes observed in accreting Galactic black hole binaries (Casares \& Jonker 2014). Multi-wavelength observations have largely ruled out strong anisotropic emission as a means of skewing luminosity estimates (Moon et al. 2011; although moderate collimation is still permitted). ULXs must therefore either host large black holes, potentially either the long-postulated yet elusive "intermediate mass" black holes $\left(M_{\mathrm{BH}} \sim 10^{2-5} M_{\odot}\right.$; Miller et al. 2004) or the massive stellar remnants $\left(M_{\mathrm{BH}} \sim 30-100\right.$ $M_{\odot}$; Zampieri \& Roberts 2009$)$ recently confirmed by LIGO (Abbott et al. 2016), or represent an exotic, highly superEddington accretion phase (Poutanen et al. 2007). In either case, they hold clues to the processes governing the formation and evolution of supermassive black holes in the early universe (Kormendy \& Ho 2013).

Since launch, the NuSTAR mission (Harrison et al. 2013) has undertaken a substantial program observing a sample of extreme ULXs, revealing the high-energy $(E>10 \mathrm{keV})$ behavior of these enigmatic sources for the first time. As one of the few sources within $\sim 5 \mathrm{Mpc}$ to persistently radiate at $L_{\mathrm{X}} \sim 10^{40} \mathrm{erg} \mathrm{s}^{-1}$ (Miller et al. 2013), NGC $1313 \mathrm{X}-1$ ( $D \sim 4$ $\mathrm{Mpc}$ ) was an important part of this program, observed in coordination with XMM-Newton (Jansen et al. 2001) to provide broadband $(\sim 0.3-30 \mathrm{keV})$ spectral coverage. These observations revealed broadband spectra inconsistent with standard modes of sub-Eddington accretion (Bachetti et al. 2013; Miller et al. 2014; similar to other ULXs observed by NUSTAR to date, Walton et al. 2014, 2015a, 2015b; Mukherjee et al. 2015; Rana et al. 2015), supporting the idea that these sources are exhibiting a super-Eddington phase of accretion. Indeed, we now know at least one of these sources is a highly superEddington neutron star (Bachetti et al. 2014).

A prediction of all super-Eddington accretion models is that powerful winds should be launched (Poutanen et al. 2007; King 2009; Dotan \& Shaviv 2011; Takeuchi et al. 2013). Robust detection of any such winds from ULXs has, however, proven challenging (Walton et al. 2012, 2013). For NGC 1313 X-1, Middleton et al. (2015b) report low-energy $(\sim 1 \mathrm{keV})$ blended atomic features that are consistent with being absorption from an ionized outflow, but the low-resolution CCD spectra considered prevented a conclusive identification as such. However, in a key breakthrough, a recent follow-up analysis by Pinto et al. (2016) utilizing the high-resolution reflection grating spectrometer (RGS) on board XMM-Newton was able to resolve this low-energy spectral structure into several discrete emission and absorption features, and found that NGC $1313 \mathrm{X}-1$ does indeed exhibit an extreme ionized outflow, potentially consisting of multiple velocity components spanning $\sim 0.2-0.25 c$.

Here, by considering the high-energy XMM-Newton and NuSTAR data available for NGC $1313 \mathrm{X}-1$, we report on a detection of an ionized iron $\mathrm{K} \alpha$ component to the ultrafast outflow (UFO) discovered by Pinto et al. (2016).

\section{OBSERVATIONS AND DATA REDUCTION}

NGC 1313 X-1 has frequently been observed in the X-ray band and is known to be a variable source, exhibiting high and low states with significantly different spectra (Feng \& Kaaret 2006; Pintore \& Zampieri 2012; Middleton et al. 2015a; M. Bachetti et al. in preparation). The majority of the X-ray observations in the archive cover the low-flux state, so we focus on these data in order to maximize the integrated signal-to-noise ratio $(\mathrm{S} / \mathrm{N})$ in the iron $\mathrm{K}$ bandpass while considering only observations with similar spectra. $X M M$-Newton, in particular, has frequently observed NGC 1313, but many of these observations are short and have $\mathrm{X}-1$ placed off-axis. The EPIC-pn detector on board XMM- 


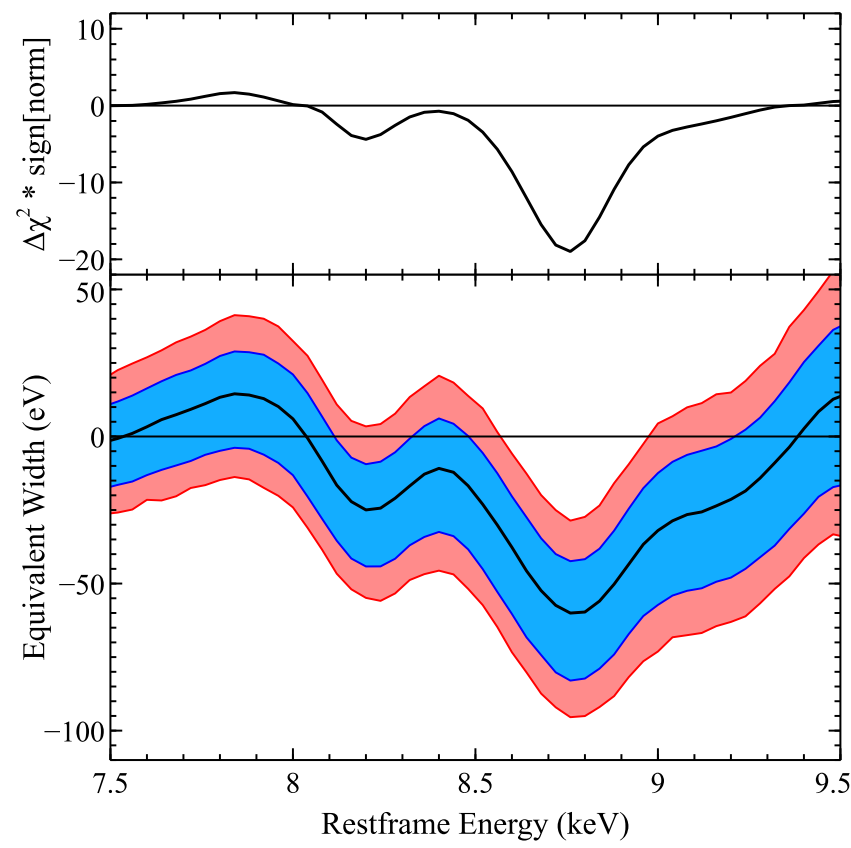

Figure 1. Zoom-in on the line-search results for the absorption feature detected in our analysis. Top: the $\Delta \chi^{2}$ improvement obtained with the addition of a narrow Gaussian line, as a function of line energy, for NGC 1313 X-1. Positive values indicate the best-fit line is in emission, and negative values indicate absorption. The feature at $\sim 8.8 \mathrm{keV}$ gives an improvement of $\Delta \chi^{2}=19.5$. Bottom: $90 \%$ (blue) and $99 \%$ (red) confidence contours for the equivalent width of the narrow line included. We find this feature at $\sim 8.8 \mathrm{keV}$ to have $\mathrm{EW}=61 \pm 24 \mathrm{eV}$. No other significant features are detected.

Newton is known to have increased background emission from copper lines at $\sim 8 \mathrm{keV}$ away from the optical axis (Carter \& Read 2007), which fall in the energy range of interest for any iron line searches given the velocities reported by Pinto et al. (2016). We therefore also limit our analysis to XMM-Newton observations where NGC $1313 \mathrm{X}-1$ was the primary target. In total, we consider the three full-orbit XMMNewton observations (OBSIDs 0405090101, 0693850501, 0693851201; note that these are the same XMM-Newton observations considered by Pinto et al. 2016), and the two long NuSTAR observations presented in Bachetti et al. (2013; OBSIDs 30002035002 and 30002035004).

The data from these observations are reduced individually, and then combined into a set of average spectra using ADDASCASPEC. In all cases, source products are extracted from a circular region of radius $40^{\prime \prime}$ since there is another X-ray source $\sim 55^{\prime \prime}$ to the south (Bachetti et al. 2013). This aperture gave the best balance between maximizing the $\mathrm{S} / \mathrm{N}$ for $\mathrm{X}-1$ and minimizing contamination from this other source. Background is always estimated from significantly larger regions of blank sky on the same detector as X-1 to ensure it is well sampled. Finally, each of the average spectra are rebinned to a minimum of 25 counts per bin to allow the use of $\chi^{2}$ minimization during spectral fitting. The following sections provide further technical details regarding our reduction of these data.

\subsection{NUSTAR}

The NUSTAR data were reduced using the standard pipeline, NUPIPELINE, part of the NuSTAR Data Analysis Software (v1.4.1). NuSTAR caldb v20150316 is used throughout. The unfiltered event files were cleaned with the standard depth correction, significantly reducing the internal background, and passages through the South Atlantic Anomaly were removed. Source spectra and instrumental responses were produced for both of the focal plane modules (FPMA/B) using NUPRODUCTS. In addition to the standard "science" data, we also extract the "spacecraft science" data following Walton et al. (2016), which in this case provides $\sim 35 \%$ of the total $360 \mathrm{ks}$ (per FPM) good exposure.

\subsection{XMM-Newton}

The XMM-Newton data were reduced with the XMM-Newton Science Analysis System (v14.0.0), following the standard prescription. ${ }^{8}$ Raw data files were cleaned using EPCHAIN for EPIC-pn (Strüder et al. 2001) and EMCHAIN for the EPICMOS detectors (Turner et al. 2001). Only single-double $($ PATTERN $\leqslant 4)$ and single-quadruple $($ PATTERN $\leqslant 12$ ) events were considered for EPIC-pn and EPIC-MOS, respectively. Periods of high background were excluded, as were events close to edge/bad pixels $(F L A G=0)$. Instrumental response files were generated with fgen and ARFGEN, and the data from the two EPIC-MOS detectors were combined together. The total good exposure is $258 \mathrm{ks}$ for EPIC-pn and $331 \mathrm{ks}$ for each EPIC-MOS unit.

\section{SPECTRAL ANALYSIS}

Our goal in this work is to search for any ionized iron $\mathrm{K} \alpha$ absorption features in the X-ray spectrum of NGC $1313 \mathrm{X}-1$ that might be associated with the UFO discovered by Pinto et al. (2016). We use XSPEC v12.8.2 (Arnaud 1996) for our spectral analysis, and unless stated otherwise parameter uncertainties are quoted at $90 \%$ confidence for one parameter of interest. Our data selection is such that we can apply a common model to all the data sets, accounting for differences in the average XMM-Newton and NUSTAR fluxes and residual cross-calibration uncertainties between the detectors by allowing multiplicative constants to float between them, fixing EPICpn to unity. The EPIC-pn and EPIC-MOS detectors agree to within 5\%, as do FPMB and FPMB, while the average XMMNewton and NuSTAR fluxes resulting from our data selection only differ by $\sim 10 \%$, which is broadly similar to the absolute cross-calibration differences seen between the two missions (Madsen et al. 2015).

We begin by constructing a simple model for the continuum. In this work, we focus on the $3-20 \mathrm{keV}$ bandpass, providing sufficient coverage to accurately model the continuum local to the iron band with a simple model, while remaining independent of the low-energy X-ray band in which the outflow was initially discovered. The spectral curvature seen from NGC $1313 \mathrm{X}-1$ over this energy range is well established (Stobbart et al. 2006; Gladstone et al. 2009; Bachetti et al. 2013), so we model the continuum as a cutoff power law. We also include neutral absorption, both from our Galaxy $\left(N_{\mathrm{H}, \mathrm{Gal}}=4.1 \times 10^{20} \mathrm{~cm}^{-2}\right.$; Kalberla et al. 2005) and intrinsic to NGC $1313 \quad(z=0.00157)$. However, given the limited bandpass considered, we are not particularly sensitive to the level of absorption seen toward NGC $1313 \mathrm{X}-1$, so we fix the intrinsic column to $N_{\mathrm{H} \text {,int }}=2.7 \times 10^{21} \mathrm{~cm}^{-2} \quad$ (Miller et al. 2013). These neutral absorption components are modeled with TBABS, adopting the abundance set of Wilms et al.

\footnotetext{
8 http://xmm.esac.esa.int/
} 
(2000) and cross-sections of Verner et al. (1996). This provides an excellent fit to the $3-20 \mathrm{keV}$ emission, with $\chi^{2} /$ degrees of freedom $=1634 / 1627$. The photon index and high-energy cutoff obtained are $\Gamma=0.96 \pm 0.07$ and $E_{\text {cut }}=5.5_{-0.3}^{+0.4} \mathrm{keV}$, and the model normalization is $(4.0 \pm 0.2) \times 10^{-4}$ ct $\mathrm{keV}^{-1} \mathrm{~cm}^{-2} \mathrm{~s}^{-1}$ (at $1 \mathrm{keV}$ ).

To search for atomic features, we follow a similar approach to Walton et al. $(2012,2013)$. We refer the reader to those works for a detailed description, but in brief, we include a narrow (intrinsic width of $\sigma=10 \mathrm{eV}$ ) Gaussian, and vary its energy across the energy range of interest in steps of $40 \mathrm{eV}$ (oversampling the XMM-Newton energy resolution by a factor 4-5). The Gaussian normalization can be either positive or negative. For each line energy, we record the $\Delta \chi^{2}$ improvement in fit resulting from the inclusion of the Gaussian line, as well as the best-fit equivalent width (EW) and its $90 \%$ and $99 \%$ confidence limits. These are calculated with the EQWIDTH command in XSPEC, using 10,000 parameter simulations based on the best-fit model parameters and their uncertainties. To be conservative, we vary the Gaussian line energy between 6.6 and $9.6 \mathrm{keV}$, corresponding to a wide range of outflow velocities extending up to $>0.25 c$ for Fe XXVI.

The results are shown in Figure 1. We find the addition of a Gaussian absorption line at $\sim 8.8 \mathrm{keV}$ provides a notable improvement to the fit. Allowing the line parameters to vary freely, we find a line energy of $E=8.77_{-0.06}^{+0.05} \mathrm{keV}$, an equivalent width of $\mathrm{EW}=-61 \pm 24 \mathrm{eV}$ (comparable to the strongest iron absorption seen from a black hole binary to date; King et al. 2012). This gives an improvement to the fit of $\Delta \chi^{2}=19.5$ for three extra free parameters. The line is consistent with being unresolved at the resolution of the XMM-Newton and NuSTAR detectors $(\sigma<0.24 \mathrm{keV})$. Assuming an association with iron, the extreme energy of this feature makes an association with either Fe XXV or Fe XXVI K $\alpha$ (6.67 and $6.97 \mathrm{keV}$, respectively) the most likely. This would imply an outflow velocity of $\sim 0.2 c$ for Fe XXVI, or an even more extreme velocity of $\sim 0.25 c$ for Fe XXV. No other features provide such a strong improvement in the fit.

In Figure 2, we show the data/model ratios for the model excluding and including this absorption line. The feature is not particularly visually prominent and would be lost in the noise for any of the detectors individually. However, the key to the statistical improvement observed is that all of the detectors show low residuals to the continuum model at the same energy. In Figure 3, we show the same line search applied excluding each of the four detectors utilized in this work in turn. In each of these cases, the improvement provided by including an absorption line at $\sim 8.8 \mathrm{keV}$ is still $\Delta \chi^{2}=13-15$. This strongly implies that this feature cannot be related to systematic effects (e.g., instrumental background), which differ for each of these detectors.

\subsection{Significance Simulations}

In order to assess the statistical significance of this potential Fe $\mathrm{K}$ absorption feature, we performed a series of spectral simulations. Using the same response and background files, and adopting the same exposure times as the real data used here, we simulated 10,000 sets of XMM-Newton (pn, combined MOS1, and MOS2) and NuSTAR (FPMA, FPMB) spectra with the FAKEIT command in XSPEC based on the best-fit cutoff power-law continuum (i.e., without any absorption feature included). Each of the simulated data sets was rebinned in the

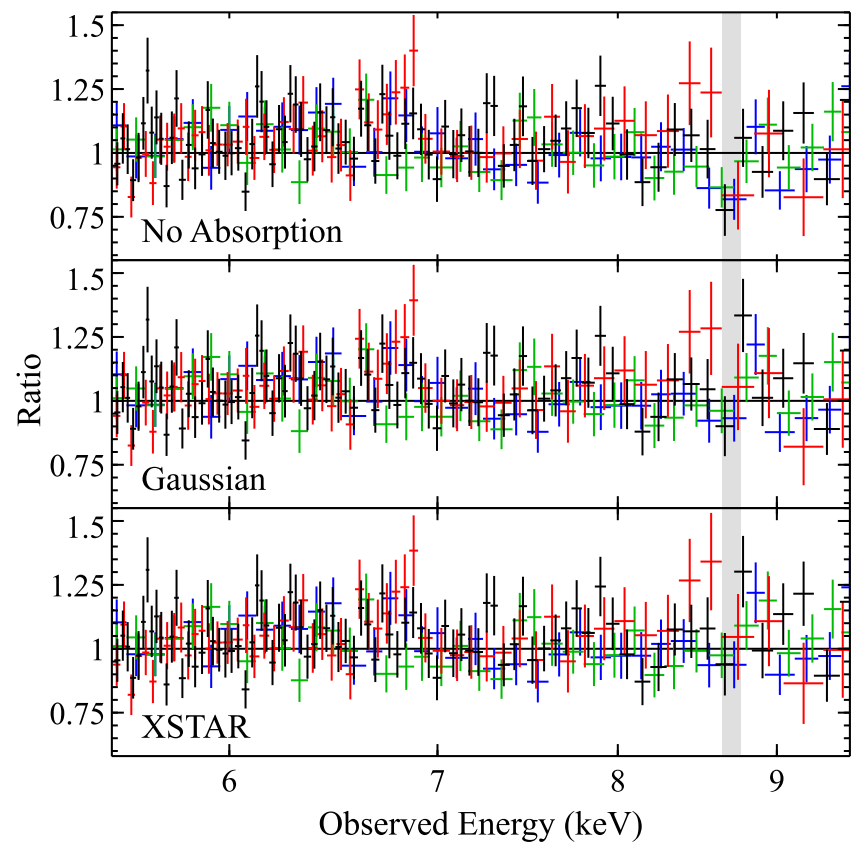

Figure 2. Data/model ratio plots for our basic continuum model (top panel), the model including a Gaussian absorption line (middle panel), and the model including a physical photoionized absorber (XSTAR; bottom panel). Data from EPIC-pn, EPIC-MOS, FPMA, and FPMB are shown in black, red, green, and blue, respectively. The feature at $\sim 8.8 \mathrm{keV}$ is indicated with the gray shaded region; all of the detectors used in this work show low residuals at the same energy, which are improved in the models including a Gaussian and an XSTAR absorption component.

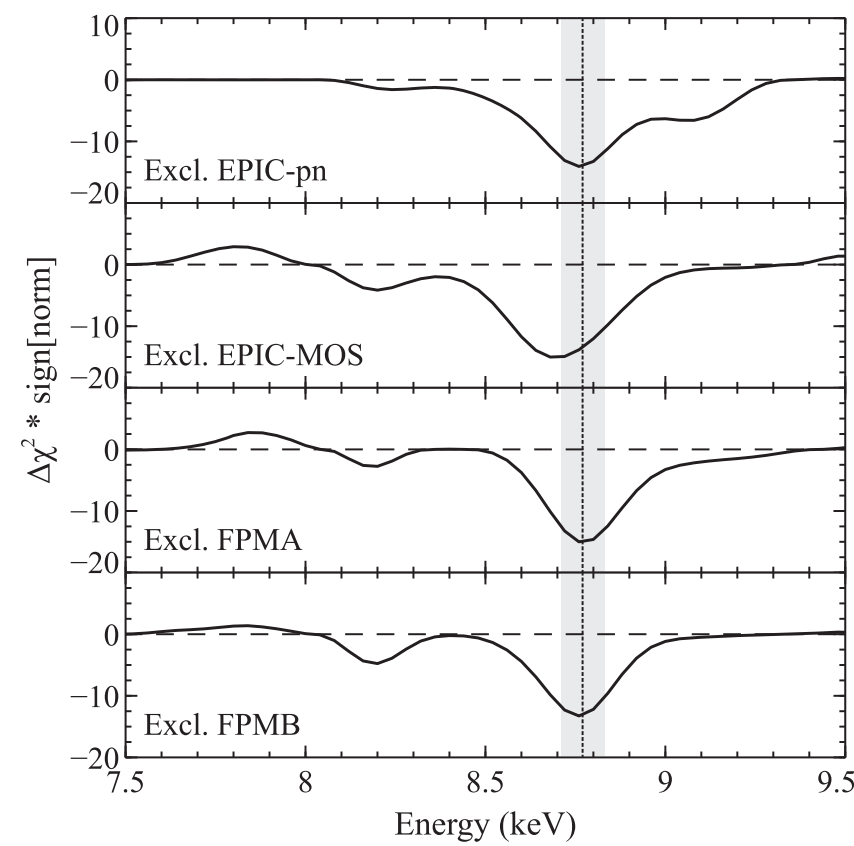

Figure 3. Results obtained repeating our line-search analysis after excluding each of the detectors utilized in turn. In all cases, the same feature is picked out. The significance is lower, as expected given the loss of $\mathrm{S} / \mathrm{N}$, but the improvement is still always $\Delta \chi^{2}=13-15$ (for 3 extra DoF). The dotted line and gray shaded region indicate the best-fit line energy and its uncertainty from the full analysis incorporating all detectors.

same manner and analyzed over the same bandpass as adopted for the real data. We then fit each of the combined data sets with a cutoff power-law continuum, and subsequently applied an identical line search as performed above (thus the number of 

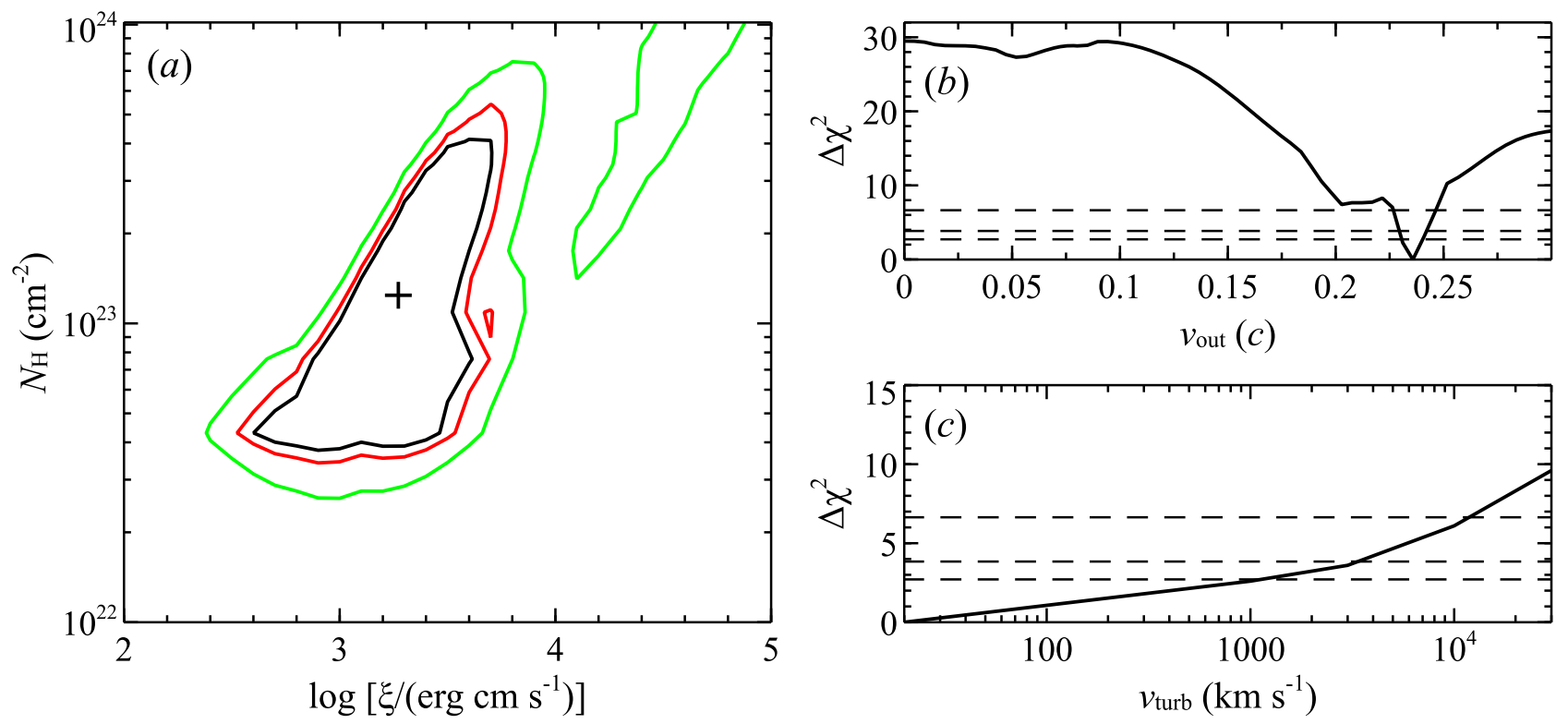

Figure 4. Confidence contours for the column density and ionization parameter (2D; panel (a)), the outflow velocity (panel (b)), and the turbulent velocity broadening (panel (c)) obtained from our photoionization modeling with XSTAR. Panel (a) shows the $90 \%, 95 \%$, and $99 \%$ contours for two parameters of interest, and the dashed lines in panels (b) and (c) show these same confidence levels for a single parameter.

energy bins searched is fully accounted for). Of the 10,000 data sets simulated, only 31 returned a chance improvement equivalent to or greater than that observed (at any energy searched), implying that the feature seen in the real data is significant at the $\sim 3 \sigma$ level. This is something of a conservative estimate; if we also require that the simulations match the exclusion trial results given above (such that the deviation is not dominated by a single detector), only 12 match the observed characteristics by chance, indicating a $\sim 99.9 \%$ detection significance. Using the velocity information from Pinto et al. (2016) as a prior would also serve to further increase the detection significance.

\subsection{XSTAR Modeling}

We model this absorption feature using a physical model for absorption by a photoionized plasma. Following Middleton et al. (2014), we construct a set of custom photoionized absorption models with XSTAR (Kallman \& Bautista 2001), using the continuum emission observed from NGC 1313 X-1 (described above) as the input ionizing continuum. These grids are calculated assuming solar abundances, an ionizing luminosity of $10^{40} \mathrm{erg} \mathrm{s}^{-1}$ (typical for NGC $1313 \mathrm{X}-1$ ), and a density of $10^{17} \mathrm{~cm}^{-3}$ (see Middleton et al. 2014). Free parameters are the ionization parameter $\left(\xi=L_{\text {ion }} / n R^{2}\right.$, where $L_{\text {ion }}$ is the ionizing luminosity between 1 and $1000 \mathrm{Ry}, n$ is the density of the plasma, and $R$ is its distance from the ionizing source; $\xi$ is calculated in units of $\mathrm{erg} \mathrm{cm} \mathrm{s}^{-1}$ throughout this work), the column density $\left(N_{\mathrm{H} \text {,ion }}\right)$, and the line of sight outflow velocity $\left(v_{\text {out }}\right)$ of the absorbing medium. We also consider a range of turbulent velocity broadening, $v_{\text {turb }}$, from 20 to $30,000 \mathrm{~km} \mathrm{~s}^{-1}$, for consistency with Pinto et al. (2016).

The addition of XSTAR to our continuum model provides a more substantial improvement to the fit than the single Gaussian feature: $\Delta \chi^{2}=29$ for three extra free parameters. This may suggest that, in addition to the feature at $\sim 8.8 \mathrm{keV}$, there are further weak features in the spectrum that are associated with the same outflow but not significantly detected individually. We find $\log \xi=3.3_{-0.5}^{+0.3}, \quad N_{\mathrm{H} \text {,ion }}=$ $\left(1.2_{-0.8}^{+2.1}\right) \times 10^{23} \mathrm{~cm}^{-2}, \quad v_{\text {out }}=0.236 \pm 0.005 c$, and $v_{\text {turb }}<$ $1000 \mathrm{~km} \mathrm{~s}^{-1}$. Although $\log \xi \sim 3.3$ is preferred, there is a degeneracy between the ionization parameter and the column density (Figure 4). This is not surprising given that the observational signature of this absorber is dominated by a single line, making it difficult to distinguish between ionization states dominated by Fe XXV $(\log \xi \sim 3.3)$ and Fe XXVI ( $\log \xi \sim 4.5$; see, e.g., King et al. 2014). The velocity contour therefore also shows two minima with similarly good fits (the second being at $v_{\text {out }} \sim 0.2 c$; Figure 4 ), corresponding to these two potential solutions.

\section{DISCUSSION AND CONCLUSIONS}

We have presented the detection of an absorption feature at $E=8.77_{-0.06}^{+0.05} \mathrm{keV}$ in the X-ray spectrum of the ULX NGC $1313 \mathrm{X}-1$, found by combining data from the XMMNewton and NuSTAR observatories. Owing to the extreme energy of this feature, and the low flux of NGC $1313 \mathrm{X}-1$, the combination of XMM-Newton and NUSTAR is particularly vital to this detection. This provides a broad bandpass, enabling robust continuum estimation both above and below the line energy, and significantly enhances the $\mathrm{S} / \mathrm{N}$ over what each observatory individually would return in commensurate exposure times. Both of these issues hindered our previous attempt to search for absorption in NGC $1313 \mathrm{X}-1$ (using XMM-Newton only; Walton et al. 2012) to the extent that this feature could not be seen. Furthermore, the combination of the different detectors on board XMM-Newton and NUSTAR allows us to effectively rule out an instrumental systematic origin, given that all the detectors utilized show consistent low residuals to the continuum emission.

Associating this feature with highly ionized iron, either Fe XXV or Fe XXVI, implies an extreme outflow velocity of $0.2-0.25 c$. Photoionization modeling marginally prefers a solution in which this absorption is dominated by Fe XXV $\left(\log \xi \sim 3.3, v_{\text {out }} \sim 0.25 c\right)$, but with the observational 
signature of this absorber being dominated by this single line there is significant degeneracy, with solutions dominated by Fe XXVI $\left(\log \xi \sim 4.5, v_{\text {out }} \sim 0.2 c\right)$ providing similarly good fits. These velocities are consistent with the UFO recently discovered by Pinto et al. (2016), that was identified through the detection of highly blueshifted absorption lines from moderately ionized material in the low-energy X-ray band, suggesting that we are seeing an iron $\mathrm{K} \alpha$ component associated with the same outflow. Ionized iron $\mathrm{K} \alpha$ absorption features associated with UFOs $\left(v_{\text {out }}>0.1 c\right)$ have been seen in several active galaxies (e.g., Tombesi et al. 2010), but never before from an X-ray binary. We note that the energy of the detected feature is just about consistent with the rest-frame energy of the Fe XXV edge at $8.83 \mathrm{keV}$. However, the data prefer the feature to be narrow, and no corresponding absorption line is seen at $6.67 \mathrm{keV}$, hence an ionized absorber at rest provides a significantly worse fit in our photoionization modeling $\left(\Delta \chi^{2} \sim 29\right)$.

Pinto et al. (2016) consider two possibilities for the outflow structure: a single zone with low-velocity broadening $\left(v_{\text {turb }}=20 \mathrm{~km} \mathrm{~s}^{-1}\right)$, and two zones, the second of which has a much higher broadening $\left(v_{\text {turb }}=10,000 \mathrm{~km} \mathrm{~s}^{-1}\right)$. The line detected here is narrow; broadening at the latter level seems to be unlikely. We therefore compare our results to the former scenario. Although there is significant degeneracy in our results (Figure 4), the absorption detected here is significantly more ionized and has a significantly larger column; Pinto et al. (2016) found $\log \xi \sim 2.3$ and $N_{\mathrm{H}} \sim 2 \times 10^{22} \mathrm{~cm}^{-2}$ for their one-zone model. The absorption detected here may thus arise in a phase of the outflow located closer to the black hole than that contributing the features detected in the RGS. The contrast between $v_{\text {out }}$ and $v_{\text {turb }}$ is larger than inferred for the UFOs in PDS456 (Nardini et al. 2015) and PG1211+143 (Pounds et al. 2003), despite the similar Gaussian line width constraints. If real, this may provide some clue to the wind geometry, implying that we might not directly view the primary acceleration region, otherwise a smaller contrast would have been expected. However, the constraint on $v_{\text {turb }}$ is ionization dependent, with the higher ionization solution allowing for $v_{\text {turb }}$ up to $10,000 \mathrm{~km} \mathrm{~s}^{-1}$, more comparable with these other cases.

This additional phase of absorption would significantly increase the total mass outflow rate $\left(\dot{M}_{\text {out }}\right)$ compared to that inferred from just the low-energy absorption alone. Combining the standard expression for $\dot{M}_{\text {out }}$ and the definition of the ionization parameter, we can estimate the kinetic luminosity of the outflow $\left(L_{\text {kin }}=1 / 2 \dot{M} v_{\text {out }}^{2}\right)$ relative to the bolometric radiative luminosity $\left(L_{\mathrm{bol}}\right)$ :

$$
\frac{L_{\mathrm{kin}}}{L_{\mathrm{bol}}} \approx 2 \pi m_{\mathrm{p}} \mu \frac{L_{\mathrm{ion}}}{L_{\mathrm{bol}}} \frac{v_{\mathrm{out}}^{3}}{\xi} \Omega C_{\mathrm{V}}
$$

where $m_{\mathrm{p}}$ is the proton mass, $\mu$ is the mean atomic weight ( $\sim 1.2$ for solar abundances), $\Omega$ is the (normalized) solid angle subtended by the wind, and $C_{\mathrm{V}}$ is its volume filling factor (or its "clumpiness"). Although some extrapolation beyond the observed bandpass is necessary, the broadband continuum models constructed by Bachetti et al. (2013) and Miller et al. (2014) imply $L_{\text {ion }} / L_{\text {bol }} \sim 0.85$. We therefore find $L_{\mathrm{kin}} / L_{\mathrm{bol}} \sim 1500 \Omega C_{\mathrm{V}}$ and $\sim 60 \Omega C_{\mathrm{V}}$ for the lower and higher ionization solutions, respectively. Thus, unless it either has a very small solid angle or a very low volume filling factor (which may be possible; King et al. 2012), the wind may dominate the energy output from NGC 1313 X-1. While both $\Omega$ and $C_{\mathrm{V}}$ are unknown, the above $L_{\mathrm{kin}} / L_{\mathrm{bol}}$ values are extreme in comparison to similar calculations for even the strongest outflows seen from sub-Eddington systems (Blustin et al. 2005; King et al. 2012, 2014; Nardini et al. 2015; Miller et al. 2016). This is consistent with the basic expectation for a superEddington accretion scenario (Poutanen et al. 2007; King 2009), as suggested by the unusual broadband X-ray spectrum observed (Bachetti et al. 2013).

The authors would like to thank the anonymous referee for their extremely timely and positive feedback, which helped improve the final manuscript. M.J.M. acknowledges support from an STFC Ernest Rutherford fellowship, C.P. and A.C.F. acknowledge support from ERC Advanced Grant 340442, and D.B. acknowledges financial support from the French Space Agency (CNES). This research has made use of data obtained with $N u S T A R$, a project led by Caltech, funded by NASA, and managed by NASA/JPL, and has utilized the NUSTARDAS software package, jointly developed by the ASDC (Italy) and Caltech (USA). This research has also made use of data obtained with XMM-Newton, an ESA science mission with instruments and contributions directly funded by ESA Member States.

\section{Facilities: NuSTAR, XMM.}

\section{REFERENCES}

Abbott, B. P., Abbott, R., Abbott, T. D., et al. 2016, PhRvL, 116, 061102 Arnaud, K. A. 1996, in ASP Conf. Ser. 101, Astronomical Data Analysis Software and Systems V, ed. G. H. Jacoby \& J. Barnes (San Francisco, CA: ASP), 17

Bachetti, M., Harrison, F. A., Walton, D. J., et al. 2014, Natur, 514, 202

Bachetti, M., Rana, V., Walton, D. J., et al. 2013, ApJ, 778, 163

Blustin, A. J., Page, M. J., Fuerst, S. V., Branduardi-Raymont, G., \& Ashton, C. E. 2005, A\&A, 431, 111

Carter, J. A., \& Read, A. M. 2007, A\&A, 464, 1155

Casares, J., \& Jonker, P. G. 2014, SSRv, 183, 223

Dotan, C., \& Shaviv, N. J. 2011, MNRAS, 413, 1623

Feng, H., \& Kaaret, P. 2006, ApJL, 650, L75

Gladstone, J. C., Roberts, T. P., \& Done, C. 2009, MNRAS, 397, 1836

Harrison, F. A., Craig, W. W., Christensen, F. E., et al. 2013, ApJ, 770, 103

Jansen, F., Lumb, D., Altieri, B., et al. 2001, A\&A, 365, L1

Kalberla, P. M. W., Burton, W. B., Hartmann, D., et al. 2005, A\&A, 440, 775

Kallman, T., \& Bautista, M. 2001, ApJS, 133, 221

King, A. L., Miller, J. M., Raymond, J., et al. 2012, ApJL, 746, L20

King, A. L., Walton, D. J., Miller, J. M., et al. 2014, ApJL, 784, L2

King, A. R. 2009, MNRAS, 393, L41

Kormendy, J., \& Ho, L. C. 2013, ARA\&A, 51, 511

Madsen, K. K., Harrison, F. A., Markwardt, C. B., et al. 2015, ApJS, 220, 8

Middleton, M. J., Heil, L., Pintore, F., Walton, D. J., \& Roberts, T. P. 2015a MNRAS, 447, 3243

Middleton, M. J., Walton, D. J., Fabian, A., et al. 2015b, MNRAS, 454, 3134

Middleton, M. J., Walton, D. J., Roberts, T. P., \& Heil, L. 2014, MNRAS, 438, L51

Miller, J. M., Bachetti, M., Barret, D., et al. 2014, ApJL, 785, L7

Miller, J. M., Raymond, J., Cackett, E., Grinberg, V., \& Nowak, M. 2016, ApJL, 822, L18

Miller, J. M., Raymond, J., Fabian, A. C., et al. 2004, ApJ, 601, 450

Miller, J. M., Walton, D. J., King, A. L., et al. 2013, ApJL, 776, L36

Moon, D.-S., Harrison, F. A., Cenko, S. B., \& Shariff, J. A. 2011, ApJL, 731, L32

Mukherjee, E. S., Walton, D. J., Bachetti, M., et al. 2015, ApJ, 808, 64 Nardini, E., Reeves, J. N., Gofford, J., et al. 2015, Sci, 347, 860

Pinto, C., Middleton, M. J., \& Fabian, A. C. 2016, Natur, 533, 64

Pintore, F., \& Zampieri, L. 2012, MNRAS, 420, 1107

Pounds, K. A., Reeves, J. N., King, A. R., et al. 2003, MNRAS, 345, 705

Poutanen, J., Lipunova, G., Fabrika, S., Butkevich, A. G., \& Abolmasov, P. 2007, MNRAS, 377, 1187

Rana, V., Harrison, F. A., Bachetti, M., et al. 2015, ApJ, 799, 121 
Stobbart, A.-M., Roberts, T. P., \& Wilms, J. 2006, MNRAS, 368, 397

Strüder, L., Briel, U., Dennerl, K., et al. 2001, A\&A, 365, L18

Swartz, D. A., Ghosh, K. K., Tennant, A. F., \& Wu, K. 2004, ApJS, 154, 519

Takeuchi, S., Ohsuga, K., \& Mineshige, S. 2013, PASJ, 65, 88

Tombesi, F., Cappi, M., Reeves, J. N., et al. 2010, A\&A, 521, A57

Turner, M. J. L., Abbey, A., Arnaud, M., et al. 2001, A\&A, 365, L27

Verner, D. A., Ferland, G. J., Korista, K. T., \& Yakovlev, D. G. 1996, ApJ, 465,487

Walton, D. J., Harrison, F. A., Bachetti, M., et al. 2015a, ApJ, 799, 122

Walton, D. J., Harrison, F. A., Grefenstette, B. W., et al. 2014, ApJ, 793, 21
Walton, D. J., Middleton, M. J., Rana, V., et al. 2015b, ApJ, 806, 65

Walton, D. J., Miller, J. M., Harrison, F. A., et al. 2013, ApJL, 773, L9

Walton, D. J., Miller, J. M., Reis, R. C., \& Fabian, A. C. 2012, MNRAS, 426, 473

Walton, D. J., Roberts, T. P., Mateos, S., \& Heard, V. 2011, MNRAS, 416,1844

Walton, D. J., Tomsick, J. A., Madsen, K. K., et al. 2016, ApJ, in press (arXiv:1605.03966)

Wilms, J., Allen, A., \& McCray, R. 2000, ApJ, 542, 914

Zampieri, L., \& Roberts, T. P. 2009, MNRAS, 400, 677 